\title{
Study Category
}

National Cancer Institute

\section{Source}

National Cancer Institute. Study Category. NCI Thesaurus. Code C90461.

The classification of the study. 